\title{
The incremental plasticity condition application for pure bending of a curved bar (Golovin problem)
}

\author{
$S$ Kalashnikov ${ }^{1,2, *}, E$ Gurova $^{1}, S$ Kalinovsky $^{1}$ \\ ${ }^{1}$ Volgograd State Technical University, 28 Lenin Ave., Volgograd, 400005, Russia \\ ${ }^{2}$ Central research and design Institute of the Ministry of construction and housing and communal \\ services of the Russian Federation, 29 Vernadsky Ave., Moscow, 119331, Russia
}

\begin{abstract}
The limiting elastic state of a bar with large curvature under pure bending is considered on the basis of the elasticity theory exact solution. As a criterion for the limiting state, the gradient plasticity condition is used, which determines the yield onset in an inhomogeneous stress state. Analytical expressions for calculating the corresponding stresses are obtained. The results comparison with a simplified solution is given.
\end{abstract}

\section{Introduction}

The theory of strength by its purpose in the calculation of structural elements is aimed at achieving the main goal: ensuring strength and operational safety while reducing material consumption. The problem solution is provided by a numerical comparison of the objectively obtained strength characteristics of the material with the calculated values of the stress state in hazardous sections. This approach does not take into account the possible types of the elements and structures' real stress state. The experimental data analysis [1-5] shows that the yield point of the material, determined from experiments on simple tension, does not allow to reliably solve the strength problems with a non-uniform stress distribution.

This work is a continuation of [6], where the plasticity criterion is used for an elasticplastic isotropic material with a yield area (such as building steel). Its application leads to an analytical expression that makes it possible to calculate the corresponding stresses and loads. The authors are not aware of other works leading to the analytical solution.

\section{Formulation of the problem and method of solution}

Let us consider a pure bend of a bar, the axis of which is outlined in a circle. In a polar coordinate system with a pole at the curvature $O$ center (Fig. 1), the stresses at any point of the cross section are determined by the formulas obtained in the framework of the exact solution of the elasticity theory problem in 1881 by the Professor of the Saint-Petersburg State Institute of Technology Kh.S. Golovin [7]:

\footnotetext{
* Corresponding author: kalashnikovsu@mail.ru
} 


$$
\begin{gathered}
\sigma_{r}=\frac{4 M}{a}\left(\frac{R_{1}^{2} R_{2}^{2}}{\rho^{2}} \ln \frac{R_{2}}{R_{1}}-R_{2}^{2} \ln \frac{R_{2}}{\rho}-R_{1}^{2} \ln \frac{\rho}{R_{1}}\right) ; \\
\sigma_{\theta}=\frac{4 M}{a}\left(-\frac{R_{1}^{2} R_{2}^{2}}{\rho^{2}} \ln \frac{R_{2}}{R_{1}}-R_{2}^{2} \ln \frac{R_{2}}{\rho}-R_{1}^{2} \ln \frac{\rho}{R_{1}}-R_{1}^{2}+R_{2}^{2}\right) ; \\
\tau_{r \theta}=0,
\end{gathered}
$$

where the following is indicated $a=\left(R_{2}^{2}-R_{1}^{2}\right)^{2}-4 R_{1} R_{2}\left(\ln \frac{R_{2}}{\rho}\right)^{2}$.

The classical formulas (1) were obtained using the assumption of the fibers pressure against each other (in contrast to the materials resistance solution, where the absence of lateral pressures is assumed). We interpret this pressure as the concept of constraining deformations from the less stressed material volumes. As a criterion for the limiting state, we use the incremental plasticity condition, which establishes the yield onset moment in an inhomogeneous stress state [8-11].

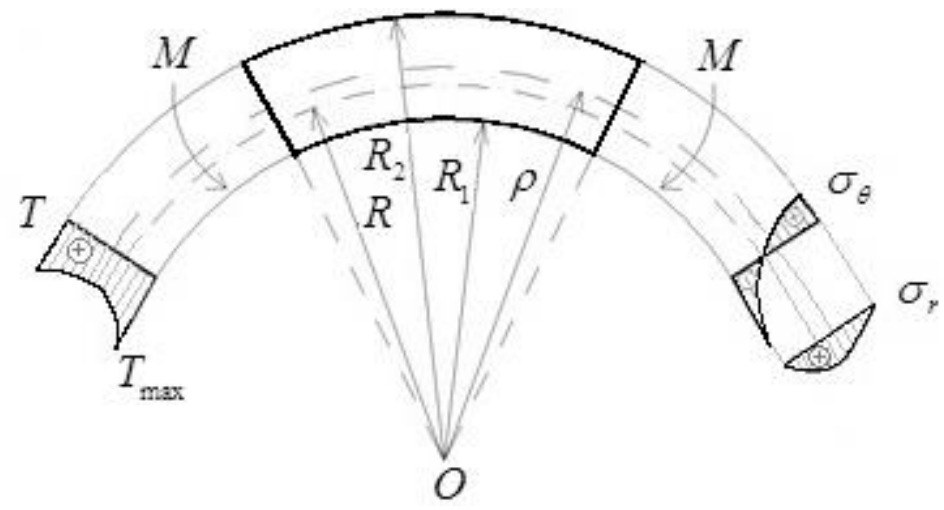

Fig. 1. Pure bending of a bar with large curvature.

The criterion is based on the above-mentioned concept of shear deformations' constraint along the sliding areas from the side of less stressed material volumes and takes into account the effect of increasing ultimate elastic stresses in inhomogeneous fields. The more inhomogeneous the stress state is, the greater the influence exerted on each other by the adjacent areas of the material. In the considered case of the one-dimensional problem, the vector-gradient is directed along the radii, therefore, the deformation is constrained in the circumferential direction. some function of the stress gradient and its derivatives with respect to coordinates. To record the incremental plasticity conditions, the form of the Tresca-SaintVenant or Huber-Mises-Hencky criteria is used, taking into account the fact that the yield stress of a material is in a certain way related to a certain function of the stress gradient and its derivatives with respect to coordinates.

Yield starts when the maximum voltage at the dangerous point reaches a certain increased value $\sigma_{\max }=\sigma_{g r}$ and the higher yield point $\sigma_{0}$. This increase will be the greater, the more inhomogeneous is the stress distribution in the vicinity of the point under consideration. At the same time, the increase cannot be unlimited, so it should be limited to some maximum $\sigma_{m}$. When using the form of the Huber-Mises-Hencky condition, the asymptotic linear fractional dependence for the intensities of shear stresses is taken as follows: 


$$
T_{g r}=T_{0}+\left(T_{m}-T_{0}\right) \cdot\left[(\operatorname{grad} T / T) /\left(\lambda_{T, g}+\operatorname{grad} T / T\right)\right]
$$

where $T_{m}$ - is the greatest of the stresses possible with a non-uniform stress state; $T_{m}=$ $1.5 T_{0} ; \lambda_{T, g}$ - is accepted for some elastic characteristic of a material that has a dimension inverse to its length. In $[11,12]$, from the experimental data [2], the numerical value $\lambda_{T, g}=$ $20,1587 \mathrm{~m}^{-1}$ is presented for construction steel.

The proposed gradient approach is implemented in solving various problems of the elements and structures' strength: in $[10,12,13]$ - in one-dimensional and two-dimensional problems of rods' bending, round plates [14, 15], torsion of round shafts [13], axisymmetric deformation of pipes and ball reservoirs [16], the rods' eccentric compression [13], tension of a plate weakened by a circular hole [17] as well as the continual problems with a singularity [18]. A feature of this condition's application, in contrast to others, is to obtain an analytical solution in the form of either a resolving equation or in the form of an algebraic expression.

Let us apply the proposed condition to the problem under consideration. In polar coordinates, the shear stress intensity

$$
T=\frac{1}{\sqrt{3}} \sqrt{\sigma_{r}^{2}+\sigma_{\Theta}^{2}-\sigma_{r} \sigma_{\Theta}+3 \tau_{r \Theta}^{2}}
$$

with substitution (1) gives:

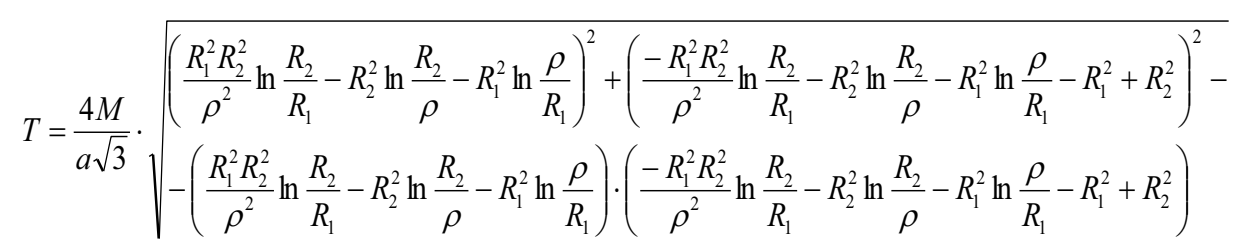

The shear stress intensity gradient modulus $\operatorname{grad} T=\sqrt{(\partial T / \partial \rho)^{2}}$ even taking into account the axisymmetry of the problem, has a cumbersome expression, which is omitted by us, and the function of the inhomogeneity measure $g=\operatorname{grad} T / T$ taking into account (2) has the form:

$$
g=\frac{1}{2 \rho} \frac{\sqrt{2 K_{2} K+2 K_{3} K_{1}-K_{3} K}}{K_{2}^{2}+K_{3}^{2}-K_{2} K_{3}}
$$

where the following is indicated

$$
\begin{gathered}
K=\frac{-2 R_{1}^{2} R_{2}^{2}}{\rho^{2}} \ln \frac{R_{2}}{R_{1}}-R_{1}^{2}+R_{2}^{2} ; \\
K_{1}=\frac{2 R_{1}^{2} R_{2}^{2}}{\rho^{2}} \ln \frac{R_{2}}{R_{1}}-R_{1}^{2}+R_{2}^{2} ; \\
K_{2}=\frac{R_{1}^{2} R_{2}^{2}}{\rho^{2}} \ln \frac{R_{2}}{R_{1}}-R_{2}^{2} \ln \frac{R_{2}}{\rho}-R_{1}^{2} \ln \frac{\rho}{R_{1}}
\end{gathered}
$$




$$
K_{3}=\frac{-R_{1}^{2} R_{2}^{2}}{\rho^{2}} \ln \frac{R_{2}}{R_{1}}-R_{2}^{2} \ln \frac{R_{2}}{\rho}-R_{1}^{2} \ln \frac{\rho}{R_{1}}-R_{1}^{2}+R_{2}^{2} .
$$

For a bar, the material of which works equally in tension and compression, regardless of the bending moment sign, the transition to the plastic state will start in the extreme fibers of a small radius at $\rho=R_{1}$. In this case

$$
g=\frac{k^{2}(6 \ln k+1)-1}{2 R_{1} \sqrt{\left(k^{2}(1-2 \ln k)-1\right)^{2}}},
$$

where through $k=R_{2} / R_{1}$ the relative height of the section is indicated.

Substituting (6) into (2), we obtain the calculation formula

$$
T_{g r}=T_{0}\left[1+0,5\left(\frac{k^{3}(6 \ln k+1)-k}{2 \lambda_{T, g} R_{2} \sqrt{\left(k^{2}(1-2 \ln k)-1\right)^{2}}+k^{3}(6 \ln k+1)-k}\right)\right] .
$$

The resulting two-parameter expression's analysis shows that it should be interpreted as follows: each bar with a radius $R_{2} \mathrm{c}$ relative height of the cross-section $k$ corresponds to a certain, and the only possible value $T_{g r}>T_{0}$, corresponding to the flow onset in the fiber.

In accordance with (7), for the bars with a relative section height in the range from 1.05 to 2 (i.e., the section height in this case ranges from $1 / 20$ of its outer radius of curvature to $1 / 2$ ), the following is expected to be noted: the smaller the height of the bar section, the greater the gradient effect, which ultimately leads to an increase in the "elastic" bearing capacity. This increase changes significantly with decreasing the curvature radius. The corresponding results are presented graphically in Fig. 2.

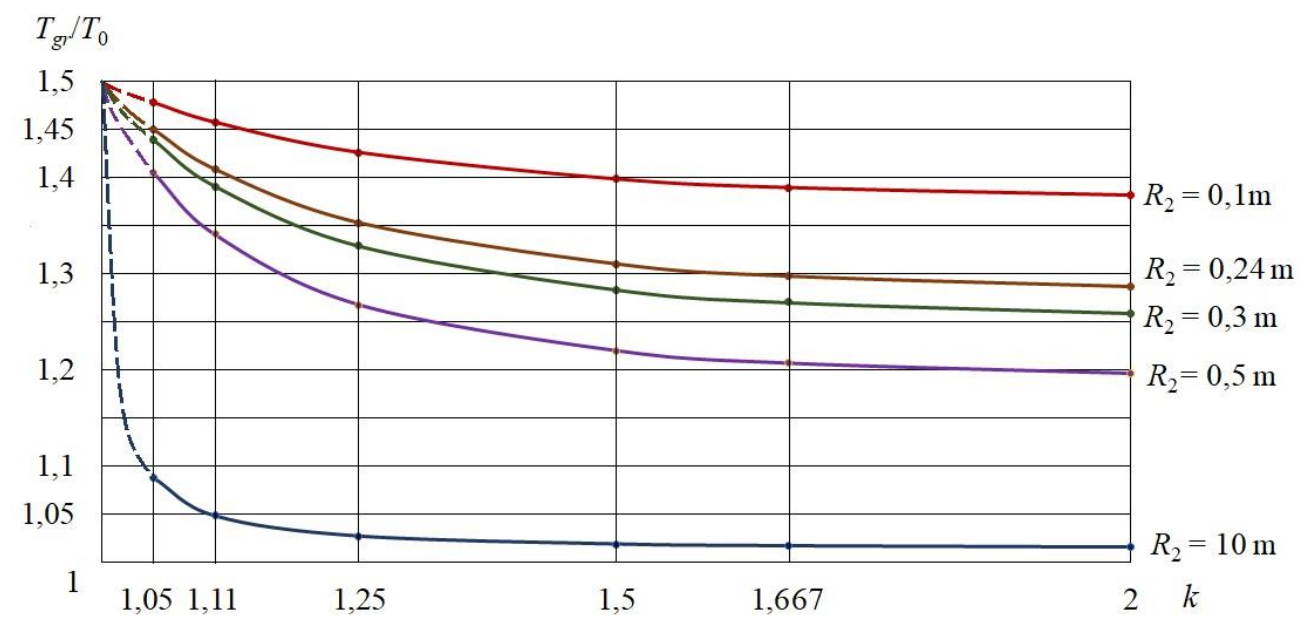

Fig. 2. Graph of change in gradient stress depending on the geometric parameters of the bar at pure bending.

Obviously, the most significant increase in the bearing capacity is manifested in the bars with the smallest cross-sectional height, but with the largest curvature radius. Overall, this increase varies widely - from $2 \%$ to $48 \%$. 
Earlier, in [6], we considered a pure bending of a bar with large curvature, when the solution was not used to determine the normal stresses in the circumferential direction Kh.S. Golovin, and the classical formula for the materials strength. It was obtained under the assumption of the absence of lateral pressures, which meant $\sigma_{r}=0$. Comparison of this formula with the solution (1) for a bar of very large curvature in [7] showed that the circumferential stresses differ by $2.5 \%$, and this is insignificant when assessing the strength. At the same time, radial stresses make up about $20 \%$ of the numerical values of the largest circumferential stresses and make a significant contribution to the magnitude of the shear stress intensity $T$. As a result, the expression was obtained:

$$
T_{g r}=T_{0} \cdot \frac{2 \lambda_{T, g}\left|R_{1} \ln k-R_{2}+R_{1}\right|-3(1-k)}{2 \lambda_{T, g}\left|R_{1} \ln k-R_{2}+R_{1}\right|-2(1-k)},
$$

in which, the modulus sign is explained by the specifics of the coordinate system, when the difference between the radius $\rho$ and the radius of the neutral axis may turn out to be negative and it should be taken in absolute value.

The comparative analysis of the values $T_{g r}$, obtained from the expressions (7) and (8) is presented in Table 1. It shows that the values of the gradient stresses obtained from (8) turn out to be even larger than those obtained by substituting the exact solution (7) into the expression of the same initial data by $0.1-1.7 \%$. The difference increases with increasing the values $k$ from 1,05 till 2, however, in the area of "reasonable" practical values of the structural elements' dimensions (tinted in the table) does not exceed 1\%.

Table 1. Relative difference $T_{g r}$ by (7) $\kappa T_{g r}$ by (8).

\begin{tabular}{|c|c|c|c|c|c|}
\hline $\boldsymbol{k}$ & $\mathbf{R . 1}$ & $\mathbf{0 . 2 4}$ & $\mathbf{0 . 3}$ & $\mathbf{0 . 5}$ & $\mathbf{1 0}$ \\
\hline 1.05 & 0.9998 & 0.9996 & 0.9995 & 0.9993 & 0.9992 \\
\hline 1.11 & 0.9993 & 0.9986 & 0.9984 & 0.9979 & 0.9989 \\
\hline 1.25 & 0.9976 & 0.9958 & 0.9953 & 0.9946 & 0.9986 \\
\hline 1.5 & 0.9944 & 0.9911 & 0.9905 & 0.9899 & 0.99816 \\
\hline 1.667 & 0.9925 & 0.9886 & 0.9879 & 0.9873 & 0.99786 \\
\hline 2 & 0.9896 & 0.9845 & 0.9837 & 0.9832 & 0.9973 \\
\hline 3 & 0.985 & 0.9772 & 0.9758 & 0.9748 & 0.9957 \\
\hline
\end{tabular}

\section{Summary}

The use of the gradient yield condition in this work, as before, leads to the analytical formulas that give satisfactory results. The bar curvature determines more significant manifestations of gradient effects compared to the pure bending of straight bars.

Comparison of the results obtained from the exact and approximate solutions indicates an insignificant difference in determining the limiting elastic state of a curved bar.

\section{References}

1. V.A. Baldin, Structural Mechanics and Analysis of Constructions 1, 29-31 (1977)

2. V.A. Baldin, V.N. Potapov, A.A. Fadeev, Structural Mechanics and Analysis of Constructions 5, 23-26 (1982)

3. A.A. Fadeev, Institute Works. Research on Strength of Elements for Metal Building Structures, 85-91 (1982) 
4. F. Campus, Bull. de la Classe des Sciences de l'Academie R. de Belgique Série. 5(49), 303-314 (1963)

5. N.M. Dehousse, Bull. de la Classe des Sciences de l'Academie R. de Belgique Série. 5(48), 329-334 (1962)

6. S.Yu. Kalashnikov, E.V. Gurova, S.A. Kalinovsky and R.Kh. Kuramshin, IOP Conference Series: Materials Science and Engineering. International Scientific Conference «Construction and Architecture: Theory and Practice of Innovative Development» (CATPID-2020) 913 (2020) Doi:10.1088/1757-899X/913/2/022008

7. V.I. Samul', Fundamentals of the theory of elasticity and plasticity ("Higher school", Moscow, 1982)

8. G.A. Geniev, S.Yu. Kalashnikov, Institute Works. Investigations in Structural Mechanics, Central Res. (Institute of Building Structures Publ., Moscow, 1985)

9. G.A. Geniev, S.Yu. Kalashnikov, Struct. Mech. and Analysis of Constr. 6, 12-15 (1988)

10. G.A. Geniev, S.Yu. Kalashnikov, On the Plotting of Incremental Plasticity Conditions (VNIIIS, Moscow, 1984)

11. S.Yu. Kalashnikov, Trudy NGASU 6, 142-148 (2003)

12. S.Yu. Kalashnikov, A.V. Levin, Dep. at Deposited Manuscript 8382, 10 (1988)

13. S.Yu. Kalashnikov, Dep. at Deposited Manuscript 5015, 16 (1984)

14. S.Yu. Kalashnikov, Numerical Methods to Solve the Problems of Elasticity and Plasticity Theory: Proceed. of XVIII Inter-Republic Conf. Kemerovo (Nonparel Publ., Novosibirsk, 2003)

15. S.Yu. Kalashnikov, University News North-Caucasian Region - Technical Sciences ser. 5, 169-174 (2003)

16. S.Yu. Kalashnikov, University News North-Caucasian Region - Technical Sciences series 3, 44-48 (2003)

17. S.Yu. Kalashnikov, E.V. Gurova, R.Kh. Kuramshin, A.V. Starov, IOP Conf. Series: Materials Science and Engineering 456, 012111 (2018) DOI: 10.1088/1757$899 \mathrm{x} / 456 / 1 / 012111$

18. S.Y. Kalashnikov, E. Gurova, R. Kuramshin, V. Kharlanov, Materials Science Forum 974, 620-26 (2019) https://doi.org/10.4028/www.scientific.net/msf.974.620 